\title{
Acercamiento a las tres traducciones castellanas del siglo XV de la Ética nicomaquea de Aristóteles
}

Approach to the Three $15^{\text {th }}$ Century Castilian Translations

of Aristotle's Nicomachean Ethics

\author{
Salvador Cuenca Almenar \\ GREMI-UJI / Universitat de València \\ salvador.cuenca@uv.es
}

\begin{abstract}
RESUMEN: El objetivo de este artículo es presentar las tres traducciones castellanas del siglo XV de la Ética nicomaquea de Aristóteles. Mediante la contraposición de tres fragmentos significativos de cada una de ellas, contrastaremos tres aspectos de sus diferentes soluciones traductológicas, a saber: la fidelidad a los antropónimos griegos, la precisión en la vulgarización de los conceptos filosóficos y la claridad en la exposición de la doctrina aristotélica.
\end{abstract}

ABSTRACT: The aim of this paper is to present the three known $15^{\text {th }}$ century Spanish translations of Aristotle's Nicomachean Ethics: Through the comparison of three significant fragments in each of these, we will contrast three aspects of his different translation solutions, namely: loyalty to the Greek anthroponyms, precision in the popularization of philosophical concepts and clarity of the explanation of Aristotelian doctrine.

Palabras ClaVE: Aristóteles, Ética, España, siglo XV, traducción.

KEY WORDS: Aristotle, Ethics, Spain, $15^{\text {th }}$ Century, Translation

RECIBIDO: 27 de enero de 2015 - ACEPTADO: 3 de septiembre de 2015.

A lo largo de los siglos XV y XVI, la ética fue la disciplina filosófica donde se produjo la intersección entre los curricula de los studia humanitatis y de las universidades escolásticas. En el Quattrocento, como es sabido, se reservaba el uso del término "humanista" para referirse a los profesores y estudiantes de studia humanitatis, a saber: poesía, gramática, retórica, historia y moral. ${ }^{1}$ Por tanto, la ética o filosofía moral era una de las cinco materias que conformaban el plan de estudios de los humanistas y, paralelamente, una de las asignaturas impartidas en las facultades de filosofía dominadas por la escolástica. ${ }^{2}$

Por ende, la lucha por el control de la filosofía moral concentró los esfuerzos de humanistas y escolásticos, es decir, la materia se convirtió en un campo de batalla intelectual donde pudieron medir sus fuerzas ambas perspectivas sobre el mundo, el lenguaje y la vida. Las traducciones de

\footnotetext{
${ }^{1}$ Cf. Kristeller 1979.

${ }^{2}$ Cf. Lines 2007, pp. 304-306.
} 
los textos griegos fundamentales para la disciplina representaron un aspecto más de la pugna por dos motivos: uno de forma y el otro de fondo. El primero concernía al tipo de traducción y a la forma de la lengua de llegada. El segundo implicaba una elección del contenido filosófico que se debía disponer en la versión trasladada. ${ }^{3}$

El objetivo de este artículo es presentar un detalle de esta pugna librada entre escolásticos y humanistas a través de la presentación de las tres traducciones al castellano del siglo xv de la Ética nicomaquea de Aristóteles:

1) El compendio escolástico (texid BETA 1294). ${ }^{4}$

2) La traducción del príncipe de Viana (texid BETA 1401). ${ }^{5}$

3) El Ms. 10268, BNE, Madrid (texid BETA 1902).

Antes de presentar los tres textos, confesaremos que el único mérito de este trabajo reside en contrastar, por primera vez para la crítica actual, algunos de sus fragmentos más conflictivos y sus diversas soluciones traductológicas, dado que el Ms. 10268 no ha sido estudiado aún por los eruditos modernos. El valor de la primicia, por tanto, envuelve al presente artículo.

Con el fin de fundamentar los tres fragmentos elegidos para realizar el contraste y entender su contenido e importancia, expondremos sucintamente el contexto de la recepción de la Ética aristotélica en la España tardomedieval. Recordemos que las cortes hispánicas del Cuatrocientos fomentaron la realización de traducciones de los clásicos para un público laico y culto, cuya formación moral ya no se basaba únicamente en la doctrina cristiana, sino también en la sabiduría de los modelos grecolatinos. ${ }^{6}$ De este modo se gestaba el enfrentamiento entre los modelos de santidad cristiana y aquellos de la civilización clásica, en tanto que fundamento textual del humanismo.?

La Ética nicomaquea ofrecía uno de estos catálogos de modelos de humanidad clásica, necesario para reflexionar sobre cómo actuar en una

\footnotetext{
${ }^{3}$ Cf. Lines 2002.

${ }^{4}$ Cf. Cuenca 2012.

${ }^{5}$ Cf. Heusch 1993 y Fernández 2002.

${ }^{6}$ Cf. Lawrance 1982.

${ }^{7}$ Cf. Pagden 1975, pp. 287-313.
} 
Europa que percibía las primeras grietas estructurales en el edificio doctrinal eclesiástico, debidas a la dilatación causada por nuevas geografías (Colón y Américo Vespuccio), cosmologías (Copérnico) y anatomías (Vesalio y Harvey). ${ }^{8}$ Sin embargo, la Ética nicomaquea no es una obra monolítica y sufrió diversos avatares desde su compilación hasta el Cuatrocientos hispánico. El texto en cuestión es el fruto de la recopilación de las notas de trabajo con las que Aristóteles (384-322 a. C.) preparaba sus lecciones de ética, cuyo primer editor fue Andrónico de Rodas entre el 40 a. C. y el 20 a. C.

La recepción en el occidente latino medieval de la Ética nicomaquea fue posterior a la de los libros de lógica, física y metafísica, aunque más duradera. Antes de 1150 Burgundio da Pisa tradujo lo que se conoce como Ethica Vetus, Ethica Nova y Ethica Borghesiana, traducción fragmentaria o, por lo menos, conservada de manera incompleta hasta nuestros días. Entre 1246 y 1247 Grosseteste tradujo la primera versión que nos ha llegado completa. Según E. Franceschini, entre 1259 y 1266 Moerbecke realizó una revisión del texto de Grosseteste; ${ }^{9}$ sin embargo, R. Gauthier refuta que sea una verdadera revisión. ${ }^{10}$ Adentrándonos ya en el siglo XV, debe destacarse que en 1417 el canciller florentino Leonardo Bruni dedicó al Papa Martín V su traducción, mientras que entre 1455 y 1459 Gianozzo Manetti realizó su traslación durante su estancia en la corte napolitana de Alfonso el Magnánimo. Por último, en 1478 Giovanni Argyropoulos dedicó su traducción al Papa Pío IV. ${ }^{11}$

Sintetizados así los avatares de la Ética nicomaquea, pasemos ya a la comparación de los rasgos esenciales de algunos fragmentos de las tres traducciones castellanas del siglo xv. El procedimiento será el siguiente: presentaremos las características fundamentales de cada traslación, para comparar después cómo se vierten en cada una tres fragmentos distintos de la Ética nicomaquea, antes de finalizar con unas conclusiones provisorias, que podrán servir de futuras líneas de investigación.

El primer fragmento nos mostrará cómo se enfrentan cada una de las tres traducciones al problema de trasladar los antropónimos griegos, en concreto, el nombre de Pericles (1140b); el segundo, a la cuestión de

\footnotetext{
${ }^{8}$ Cf. Ronzón 2012, pp. 217-235.

${ }^{9}$ Cf. Franceschini 1976.

${ }^{10}$ Cf. Gauthier 1974, p. CX.

${ }^{11}$ Cf. Lines 2002, pp. 49-51.
} 
verter el esquema aristotélico de que la virtud es un punto medio entre dos vicios, en particular, la virtud de la indignación entre la envidia y la malignidad (1108b); y el tercero, al modo de traducir las definiciones halladas por el Estagirita mediante la caracterización de sus propiedades esenciales, como la inutilidad esencial de la sabiduría (1141a). Dado que el objetivo del presente artículo es presentar los tres textos en contraste, no desarrollaremos en profundidad sus implicaciones filosóficas y nos limitaremos a apuntar meras apreciaciones formales.

\section{El Compendio escolástico}

Empecemos, pues, con el Compendio escolástico, que hemos datado alrededor de 1463 y 1464, en la publicación de la edición crítica de la versión catalana (BITECA 1405). ${ }^{12}$ Se conoce a través de un manuscrito catalán y de los siguientes códices castellanos y aragoneses: A: Arxiu de la Corona d'Aragó, Ripoll 161; Ca: Biblioteca de la Universidad de Cambridge add. 8275; Co: Biblioteca de la Universidad Complutense, Valdecilla 152; E: Biblioteca del Monasterio del Escorial K. II. 13; M1: Biblioteca Nacional, Madrid, 4514; M2: Biblioteca Nacional, Madrid, 6710; M3: Biblioteca Nacional, Madrid, 7076; M4: Biblioteca Nacional, Madrid, 1204; O: Bodleian Library, Oxford, Span. d. 1; Vat: Biblioteca Vaticana, Ottoboniano Latino 2054. ${ }^{13}$ El estudio de sus diferentes tipos de errores nos ha permitido dividirlos de la siguiente manera: la subfamilia $\left[\alpha_{\mathrm{i}}\right]$ incluye a los códices E, M1 y M3, mientras que la subfamilia $\left[\alpha_{\mathrm{ii}}\right]$ a Ca, M4 y Vat. El manuscrito Co estaría emparentado también con la familia $[\alpha]$, compartiendo errores con sus dos subfamilias. Por otro lado, la familia $[\beta]$ integraría a A, M2 y O. ${ }^{14}$ Hay que añadir que, aunque no posean valor ecdótico, las lecciones equipolentes han servido para corroborrar estos parentescos.

Por lo que respecta al aspecto lingüístico, los rasgos aragoneses del compendio se aprecian, sobre todo, en los manuscritos de la familia

${ }^{12}$ Cf. Cuenca 2012.

13 Para la descripción de las características materiales de los códices, cf. Cuenca 2012, pp. 25-35.

${ }^{14}$ Para un estudio de las relaciones y de las familias de los manuscritos castellanoaragoneses, cf. Cuenca (en prensa). 
$\left[\alpha_{\mathrm{i}}\right]$, en especial E y M3, que presentan características de la lengua de la Zaragoza del siglo xv: la -y- antihiática de "seyer" o "veyer", la conservación del grupo consonántico inicial $c l$ - de "clamar" y del $p l$ - de "pluvias", los adverbios "ensemble" o "encara", el pronombre "sende" o las formas verbales impersonales construidas a partir del tema de pasado "hubiendo", "quisiendo" o "tovido". Estos aragonesismos se encuentran también en A, un manuscrito de la familia $[\beta]$.

Dejaremos las cuestiones sobre las variedades lingüísticas del compendio para un trabajo futuro y subrayaremos que el texto muestra las características de la translatio medieval, como son los latinismos léxicos y trasliteraciones del griego, como "eutrapulos" o "bomoloco", diversos sinónimos empleados para traducir al vulgar un único concepto clásico, como "agrestes e duros" para trasladar ó cimiento de los nombres propios griegos: "Palomides" por Polidamas o "Peridea" por Pericles.

Analicemos ahora el primero de los tres fragmentos que contrastamos, donde podemos percibir que el Compendio no traduce de manera demasiado inteligible el contenido de las reflexiones aristotélicas sobre la virtud de la prudencia, cuyo paradigma político, según la Ética nicomaquea (1140b) fue Pericles. Dada la dificultad de lectura, transcribiremos el fragmento concreto que nos interesa comentar:

Los prudentes son dichos propriamente aquellos por excelencia que aconsejan a sí mismos e a los otros, e aquestos en griego son dichos Peridea. E tales son los buenos regidores de la policía e governadores de las çibdades.

En este fragmento, perteneciente al capítulo cuarto del sexto libro del Compendio (Ms. Oxford, Bodl. D. 1, f. 74r, 1. 22), podemos apreciar cómo se resume toscamente la doctrina aristotélica sobre la prudencia hasta el punto de condensarla en una sucesión de frases de difícil comprensión. Nótese que una característica esencial de la prudencia es saber deliberar - "consejar" en castellano medieval procedente del conciliare de Grosseteste - y elegir conforme a lo deliberado, no sólo a nivel individual, sino también colectivo. El Compendio deforma, probablemente debido al desconocimiento, el nombre propio de Pericles, paradigma del prudente que sabe gobernarse a sí mismo y a los demás y lo convierte en un sustantivo abstracto que, supuestamente, designaría a los que poseen la cualidad de gobernar y gobernarse bien. 


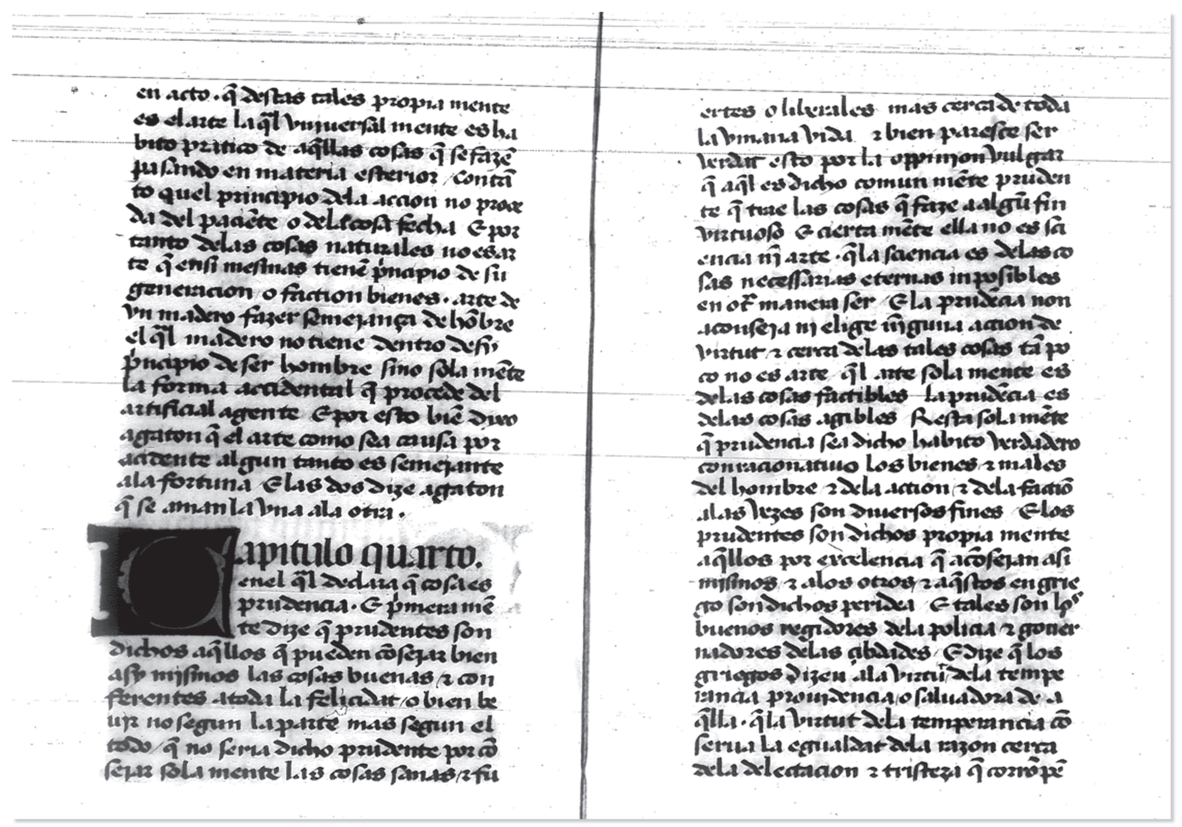

Ms. Oxford, Bodl. D. 1, ff. 73v-74r.

lomozes norio mato ö ne nene tombze elos spatmos forr filot

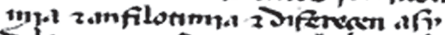
Stli m.tndmpat comola libutal,

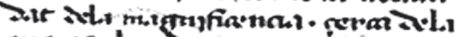

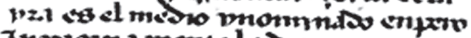
Jip piopnol mente la deztmos matr.

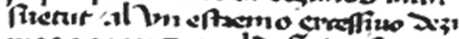
mos vzokinow il sefictuofo non tencmios nöbze fino ofle orgarmos

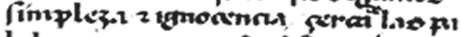

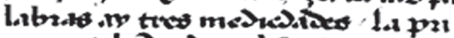
metri ez I.N hitdat elfuertestro are

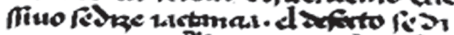

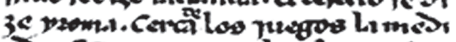

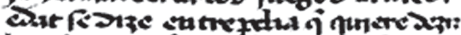

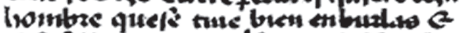

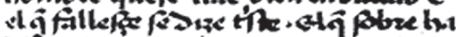

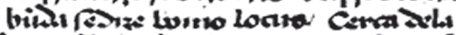

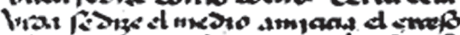

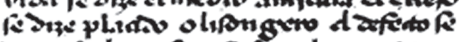

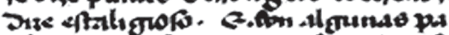

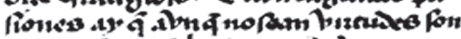

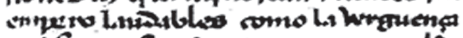

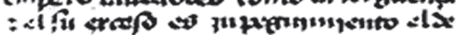

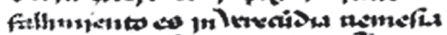

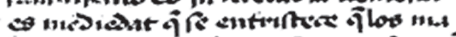

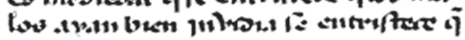
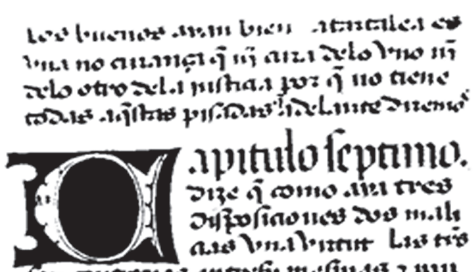

.nututo icpanco.

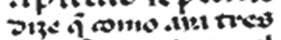
DHFofinonices oos m.1!

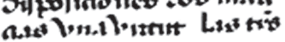

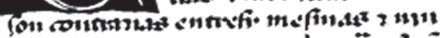

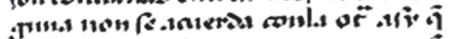

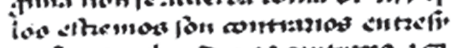

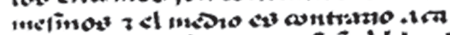

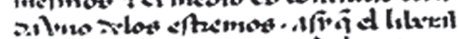

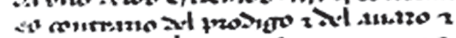

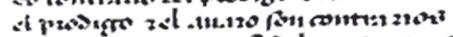

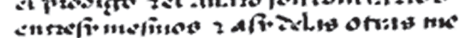

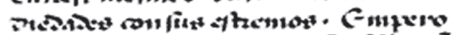

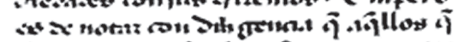

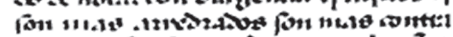

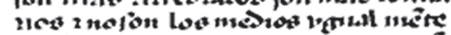

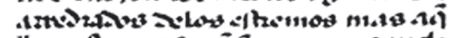

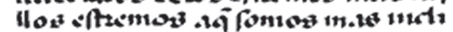

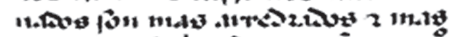

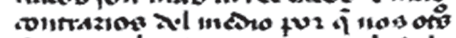

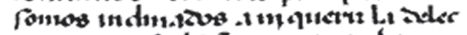

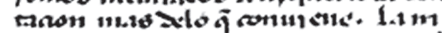

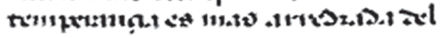

Ms. Oxford, Bodl. D. 1, ff. 35v-36r.

Todas las ilustraciones fueron proporcionadas por el autor. 
En cuanto al segundo fragmento (Ms. Oxford, Bodl. D. 1, f. 35v), debemos subrayar otra característica fundamental de la traducción compendiada, a saber, la transliteración de los términos filosóficos griegos, práctica que indica la inopia vocabularum de los traductores y la insuficiencia del latín escolástico para verter los conceptos filosóficos aristotélicos. Un indicador inequívoco de dicha inopia es la deturpación de algún concepto griego, repetido y copiado automáticamente de traducción en traducción, sin reproducir de manera fiel la conceptualización del Estagirita. Un ejemplo sangrante es el término aberrante "atartalea", hipotética traducción hispana del concepto griego epichairekakía, utilizado por Aristóteles para designar el vicio de alegrarse de que los malos prosperen. El término "atartalea" atraviesa lenguas y dialectos, ya que es utilizado tanto en catalán, como en aragonés y en castellano

Nótese también la consideración de la "nemesia", deformación de némesis (justa indignación), como pasión laudable, situada entre la envidia - caracterizada por dirigirse a la prosperidad solo de los buenos - y la aberrante "atartalea", que supuestamente se referiría a la indiferencia, sin completar que se acercaría al gozo sentido por el éxito de los malos, tal como añaden el resto de versiones.

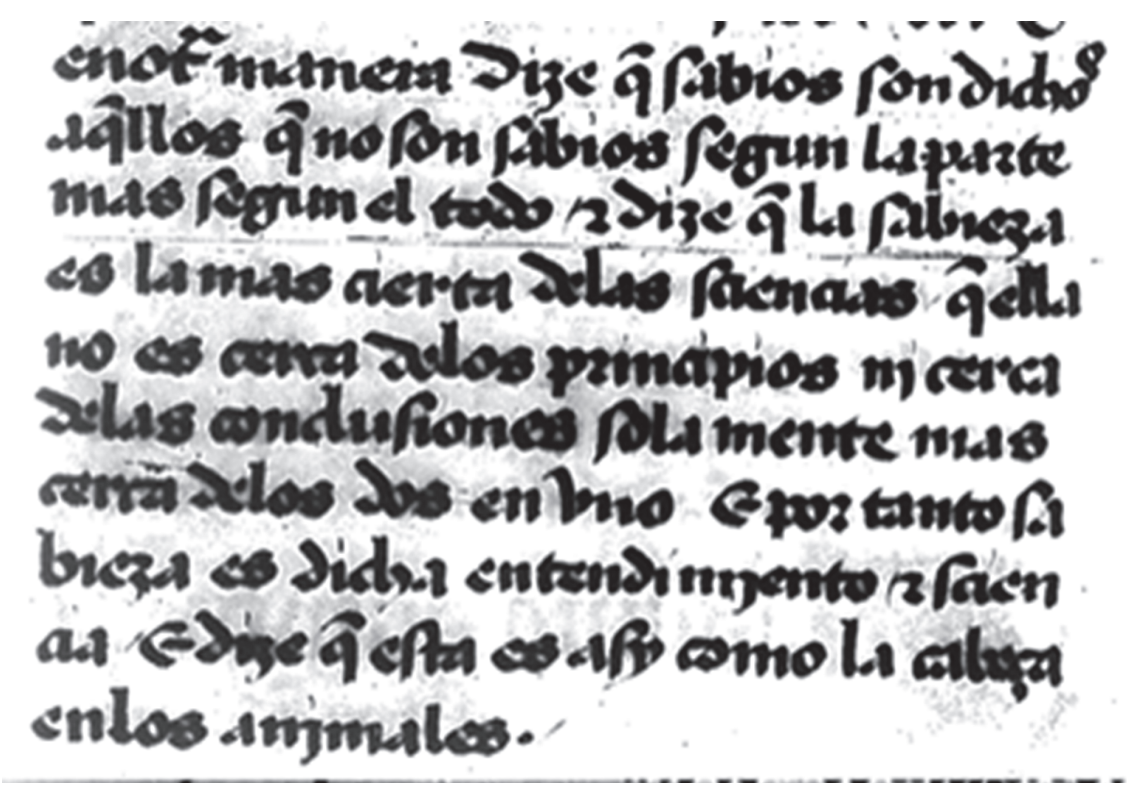

Ms. Oxford, Bodl. D. 1, fragmentos de los ff. 75v y 76v-77r. 


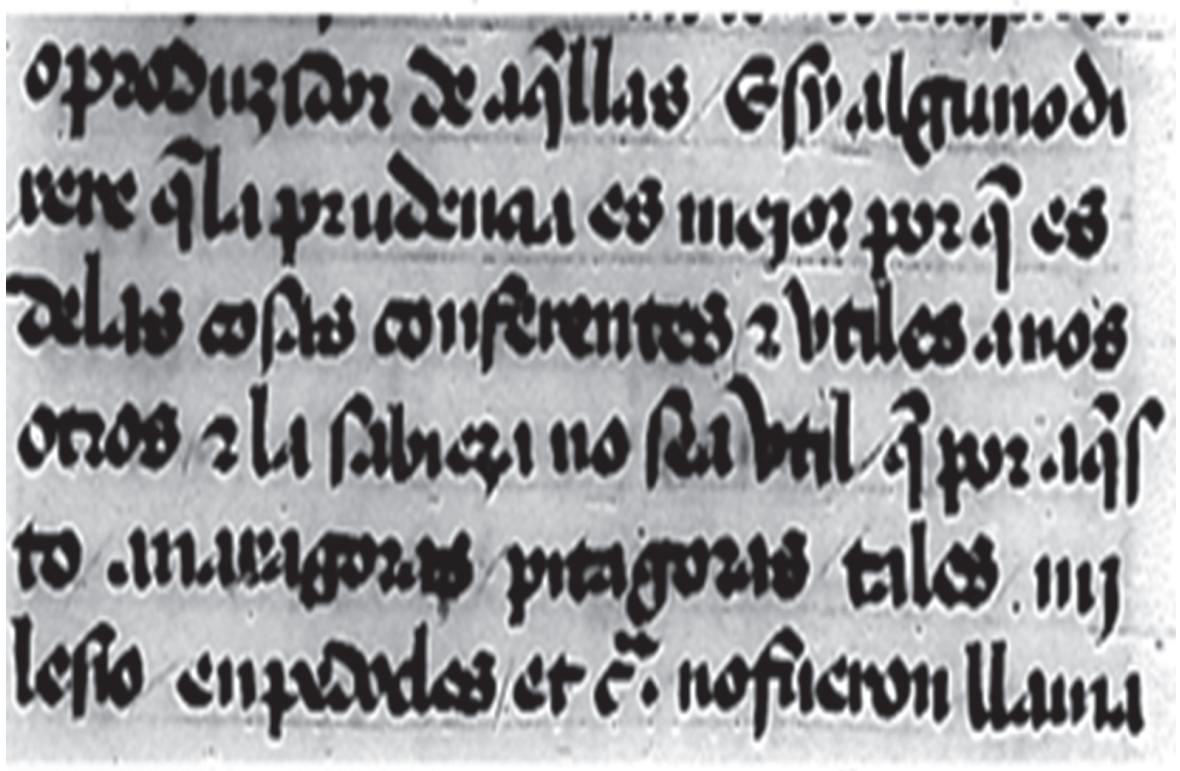

Ms. Oxford, Bodl. D. 1, fragmentos de los ff. 75v y 76v-77r.

Por lo que respecta al tercer fragmento (Ms. Oxford, Bodl. D. 1, fragmentos de los ff. 75v y 76v-77r) subrayamos que el Compendio opta por traducir la "sofía" aristotélica por sabieza, definida como hábito que englobaría tanto la capacidad del entendimiento para intuir principios, como la virtud de la "sciencia" para extraer conclusiones a partir de ellos. Notamos que esta definición es esquemática y que tan sólo explicita que la "sabieza es dicha entendimiento e sciencia", después de afirmar que no es sabio "según la parte, mas según el todo", "no cerca de los principios ni cerca de las conclusiones solamente, mas cerca de los dos en uno". Hay que explicar que la expresión "en uno" castellaniza el adverbio aragonés "ensemble".

En relación a este tercer paso, conviene resaltar que la sabieza es superior a la prudencia por su inutilidad, característica esencial de la sabiduría. Dicha inutilidad constituiría una diferencia de algunos especímenes humanos, como "Anaxágoras, Pitágoras, Tales Milesio et Empédocles", que les permitiría elevarse sobre la condición humana y las perentorias necesidades animales, para integrarse dentro de la subespecie de los sabios. Del listado de sabios anterior, subrayemos que el autor del 
Compendio añade los nombres de Pitágoras y Empédocles y que dicha adición no se encuentra ni en la traducción del príncipe de Viana ni en el Ms. BNE 10268. En cuanto al significado filosófico, podemos constatar las dificultades del Compendio para explicar que la inutilidad sea una característica esencial de la sabiduría y el criterio de demarcación entre lo valioso en sí y lo utilizable para otro fin. Recordemos brevemente, ya que dejaremos el análisis filosófico para un futuro ensayo, que el fin de los fines humanos (lo valioso en sí) es la felicidad (eudaimonía) y que su relación conceptual con la sabiduría es problemática y que varía a lo largo de la evolución intelectual del Estagirita. El problema surge de la consideración humana del fin de los fines, por la que dejaría de ser un "valor en sî" y se convertiría en un "valor para la humanidad" o "para

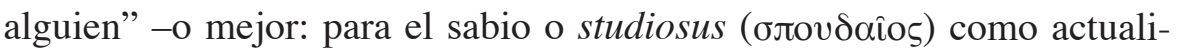
zación perfecta de las potencialidades de la especie humana.

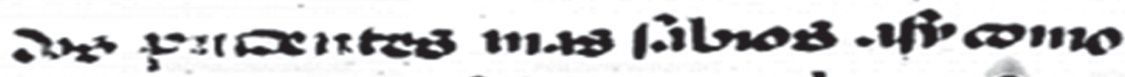

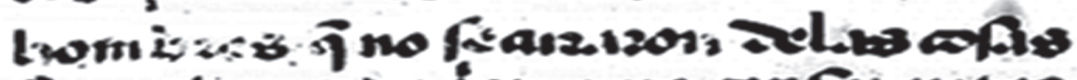

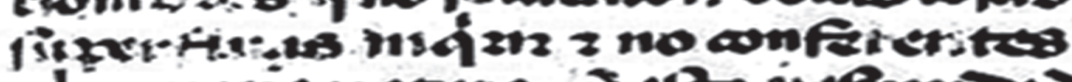

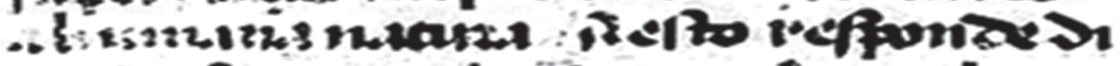

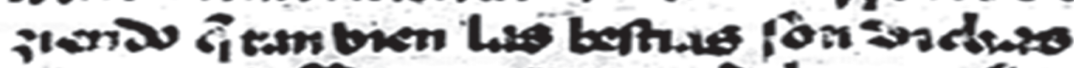

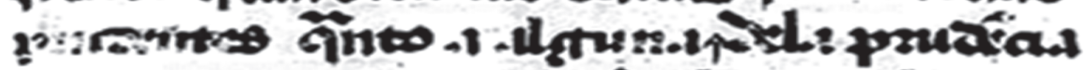

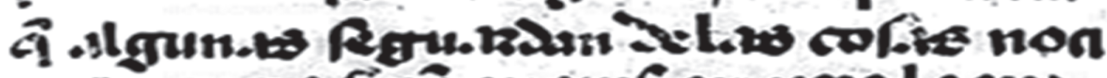

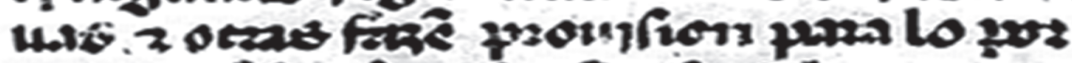

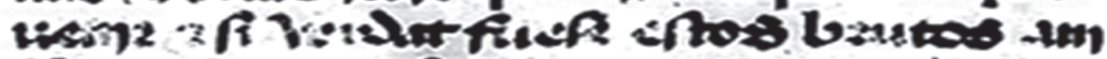

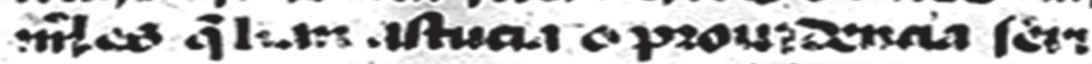

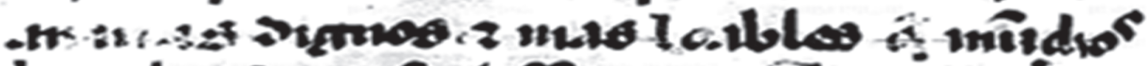

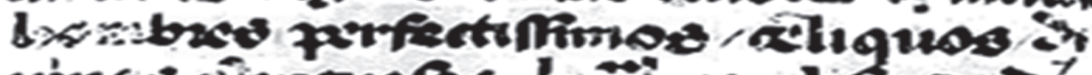

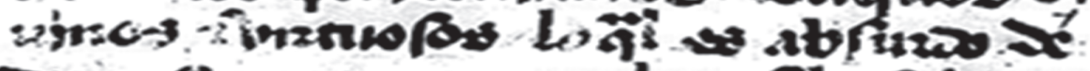

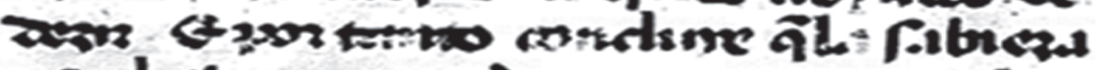

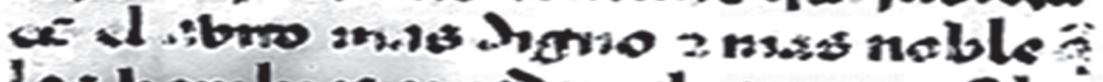

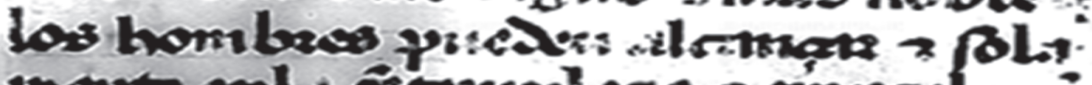

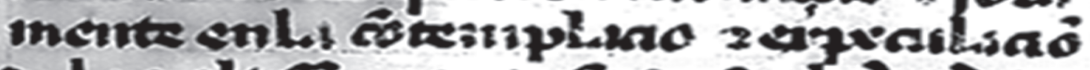

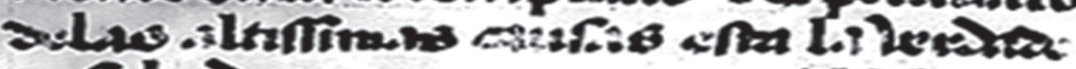

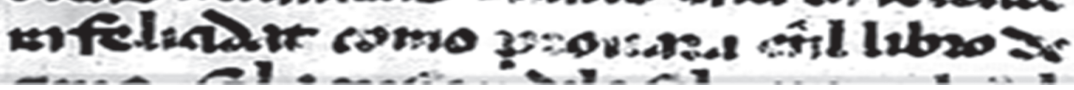

Ms. Oxford, Bodl. D. 1, fragmentos de los ff. 75v y 76v-77r. 


\section{La traducción del príncipe de Viana}

El príncipe de Viana trasladó la Ética nicomaquea del latín al castellano, durante su estancia en Nápoles, entre enero de 1457 y agosto de 1458, poco después de la muerte de su tío Alfonso el Magnánimo. El príncipe Carlos de Aragón reconoció que la finalidad esencial del trabajo fue la de congraciarse con el rey Alfonso, en cuya corte había encontrado cobijo y reposo en la huida desesperada de sus territorios navarros, tras la derrota en la batalla de Aibar infligida por las huestes de su padre enemigo en 1451. El remanso napolitano permitió a Carlos concentrar sus esfuerzos en unas labores intelectuales que podían recibir recompensas materiales por parte de su tío, en forma de territorios o de toma de partido en la lucha contra su padre, el futuro Juan II de Aragón, hermano de Alfonso.

Una de las labores intelectuales más ambiciosas fue la traducción al castellano de la Ética nicomaquea, a partir de la versión latina de Leonardo Bruni de 1417. El contexto intelectual de la corte que acogió al príncipe Carlos favorecía que los intelectuales a sueldo del Magnánimo contribuyeran a la legitimación de la conquista de Nápoles con obras literarias, como ejemplifica paradigmáticamente el De rebus ab Alphonso primo gestis del Facio. Asimismo, proliferaban los debates literarios y filosóficos, por ejemplo el de Beccadelli y Facio contra Valla acerca del códice de las Décadas de Tito Livio. ${ }^{15}$ El príncipe de Viana participó de este ambiente intelectual, en el que el regalo de una traducción de la Ética nicomaquea era muy útil para granjearse la admiración de los compañeros y el beneplácito del Rey. ${ }^{16}$

Conocemos cuatro códices del trabajo del príncipe de Viana: Lisboa, Biblioteca Nacional de Portugal IL 213; Londres, The British Library Add. 21.120; Madrid, Real Biblioteca II/2990 y Madrid, BNE, 6984. Además, hay que subrayar la importancia de la edición princeps impresa en Zaragoza en el año 1509 en el taller de Jorge Coci Alemán. Para la realización de este artículo, hemos utilizado el Ms. BNE 6984, cuyas lecciones reportamos a continuación.

En el folio 4 recto, podemos observar cómo se reformula el tópico medieval de la utilidad de las traducciones subrayando que la Ética sirve

15 Para la utilidad de los trabajos desarrollados por los humanistas, recompensados económicamente por el rey, cf. Bentley 1987, pp. 59-62.

${ }^{16}$ Cf. Cabré 2000, p. 417. 
como modelo de acción: "todos los morales se studiaron en esclarecer sus señaladas doctrinas por el común provecho que se sigue dellas". De nuevo conviene resaltar la importancia del valor de la utilidad en el Cuatrocientos, como un indicio del cambio de factores de preferibilidad introducidos por los humanistas, en detrimento de la vieja escala aristotélica y escolástica que situaba lo inutilizable, en tanto que fin de fines, en la cúspide de la escala del valor, como hemos explicado anteriormente en relación al tercer de los pasos en contraste.

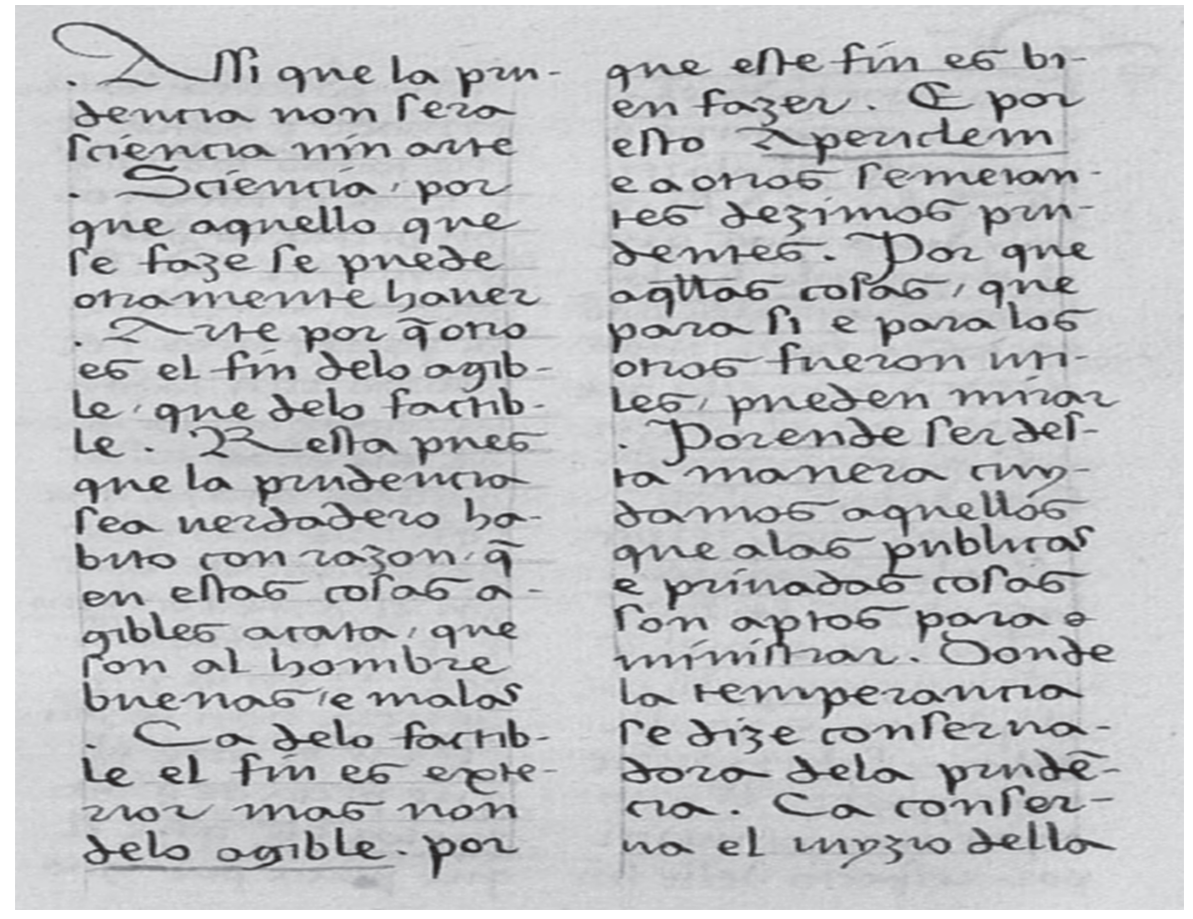

Ms. Madrid, BNE, 6984, f. 179rb 1. 2.

Transcribimos seguidamente el primero de los fragmentos a comparar (f. 179rb), con el fin de poderlos enfrentar a los textos del Compendio escolástico y del Ms. 10268 de la BNE:

E por esto a Periclem e a otros semejantes dezimos prudentes, porque aquellas cosas que para sí e para los otros fueron útiles pueden mirar. Por ende, ser desta manera cuydamos aquellos que a las públicas e privadas cosas son aptos para aministrar. 
Notamos en primer lugar que no deforma el nombre de Pericles, tal como observamos en el paso correspondiente del Compendio y como veremos en el del Ms. BNE 10268. Advertimos que el príncipe de Viana conserva la desinencia del caso latino "Periclem", cosa que nos puede guiar en una próxima investigación de las relaciones entre la traducción latina de Bruni y la vulgarización de Carlos de Aragón. En segundo lugar, percibimos que la traducción del príncipe es fiel al sentido aristotélico al utilizar la figura de Pericles de paradigma del prudente; por contra, el Compendio no acierta a verter correctamente el ejemplo paradigmático y transforma su nombre propio en peridea, un sustantivo común que se referiría aberrantemente al conjunto de los buenos gobernantes.

En el segundo de los textos en contraste, observamos que la traducción de Carlos de Aragón vierte al vulgar los nombres de los vicios y virtudes griegas, como hará el Ms. BNE. 10268 y no como el Compendio, que conserva el término griego transliterado en múltiples ocasiones. Carlos de Aragón traduce "indignación” y el Ms. BNE. 10268 "turbación”, mientras que el Compendio mantiene nemesia, como una transliteración deturpada de la némesis griega.

\begin{tabular}{|c|c|c|}
\hline 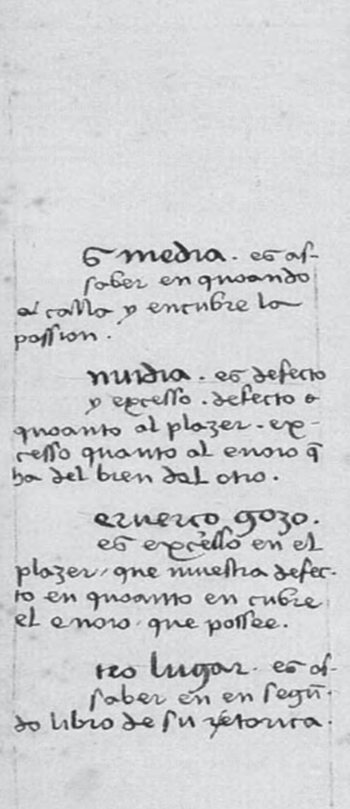 & 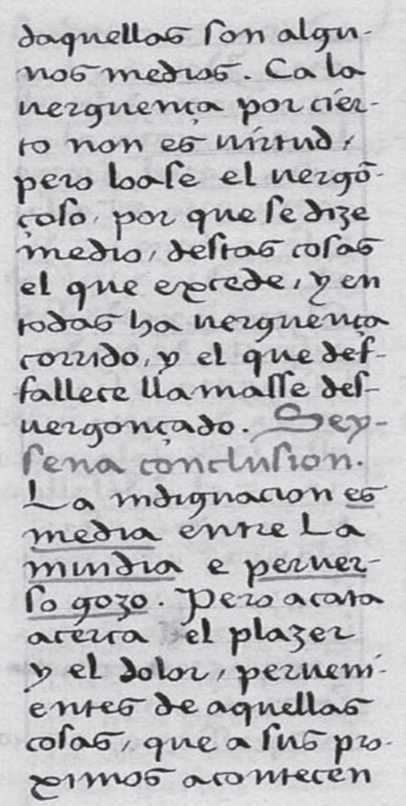 & 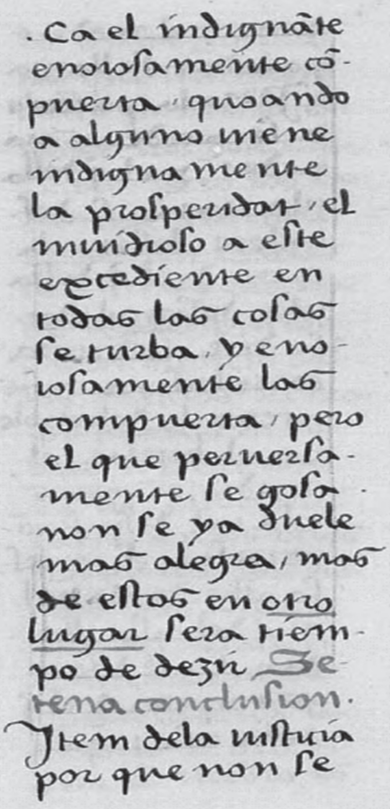 \\
\hline
\end{tabular}

Ms. Madrid, BNE, 6984, 65va-1. 14. 
Consignamos seguidamente la transcripción del fragmento que nos interesa subrayar del folio 65va:

La indignación es media entre la invidia e perverso gozo, pero acata acerca el plazer y el dolor pervenientes de aquellas cosas que a sus próximos acontecen; ca el indignante enojosamente compuerta quoando a alguno viene indignamente la prosperidat, el invidioso a este excediente en todas las cosas se turba y enojosamente las compuerta, pero el que perversamente se gosa non se ya duele mas alegra.

Indicamos el uso del sintagma perverso "gozo" para trasladar el término epichairekakía, de modo que vierte correctamente al vulgar la compleja carga semántica de esta pasión viciosa según el Estagirita. Además es coherente en la aplicación del sintagma al designar con la subordinada "el que perversamente se gosa" al que padece esta pasión viciosa. Recordemos que el Compendio reportaba la lección "atartalea" para referirse al "perverso gozo" según el príncipe de Viana y lo definía equivocadamente como un sentimiento de indiferencia respecto a la prosperidad de los demás. Adelantamos que la traducción del Ms. BNE 10268 de este complejo término también es errónea, al utilizar el sintagma "placer del mal ageno", que no se corresponde con la definición aristotélica de epichairekakía. Remarcamos otro detalle del folio consignado anteriormente: Carlos de Viana hace uso de la palabra "plazer", en oposición al "deleyte" del Ms. BNE 10268. Este detalle no tendría la más mínima importancia, de no ser que ambos términos se blandieron como estandartes de los contendientes de la controversia alphonsiana, a saber, "placer" fue la opción de Leonardo Bruni y "deleite" de Alfonso de Cartagena.

Por lo que respecta a la traducción de Carlos de Aragón del tercer fragmento de la definición de la virtud intelectual de la sabiduría (1141a-b), acentuamos la vacilación al momento de traducir uno de sus componentes esenciales, a saber, la ciencia, para el que usa el término "estimación" en el folio 181v y "sciencia" en el folio 182v. Como es sabido, la sabiduría es la virtud suprema al combinar el hábito de inteligir los principios (nous<intellectus) con el hábito de extraer a partir de ellos conclusiones mediante la demostración (epistéme<scientia). Las tres traducciones concuerdan en traducir el nous aristotélico por "entendimiento", pero divergen respecto a la epistéme, el Compendio opta por 
"sciencia", el príncipe vacila entre "estimación" y "sciencia" y el Ms. BNE 10268 prefiere "saber", la sustantivación del infinitivo.

En el folio siguiente, a saber el f. 182v, se encuentra el listado de hombres que han encarnado la sabiduría; Carlos de Viana es fiel a los ejemplos aristotélicos y solo cita a Anaxágoras y a Tales, por lo tanto no reporta la adición de Empédocles ni de Pitágoras del Compendio. Además, lee mejor los nombres propios y no los afea como el Ms. BNE 10268, que los deforma en "Amangoras e Cahalis". En la traslación principesca de la caracterización de estos sabios podemos leer que son "sapientes, mas no prudentes, porque veen a ellos los propios provechos ignorar". Por lo tanto, según este pasaje, la sabiduría es una virtud superior a la prudencia por su inutilidad, tal como se nos confirma en la frase sucesiva: "Aquellas cosas que son grandes e meravellosas e muy difíciles e divinas dizen ellos saber, e las inútiles, porque non buscan los bienes humanos". En este tercer pasaje en contraste, la traducción del príncipe de Viana se vuelve a mostrar como superior a las otras dos, tanto en la fidelidad a la letra como en la precisión del sentido.

parníular fapieme 2. fli que mamí: elo es la mas per ferta delá friemrar rer la fabrouria. Co. mene pner ol fap eme raber nom fo lameme las côth rioner matrom bren las primipi o.6 ie remer la nerdas a cezra los pon तpis. 2Nique la rabiduría poz zerso reza emen. dímémoie efímarom le arti romo rabo e rciemiza de las mas pzerwatag riémas. Erpoz nemtura reva in algumo pentalte res nar perfertala rriemra címiliola pundemia mar que la rabiduza imo que el babre fuetre la mas per fecra cofa de agllas -que en el min do rom. Jor revto fi ler rans e bue no oramemse en Las bombref le orameme en lor peré ef mas lo blámio y lo zerto riempze ef en nna maneza, ? el rabidor por to remeramse.anar el prindemte ora 


\section{El Ms. 10268 de la BNE, Madrid}

El Ms. 10268 de la BNE, olim Ii-19, contiene una traducción anónima de las Éticas de Aristóteles, tal como se lee en el tejuelo. Se trata de un trabajo del siglo XV, cuya copia fue encomendada a un escribano desconocido de nombre Morillo. El códice comprende 240 folios, estructurados por Schiff de la siguiente manera: $5+230+5$ ff. La dimensión de los folios es de $390 \times 218 \mathrm{~mm}$. Podemos observar en los fragmentos consignados a continuación que el espacio para las iniciales se dejó en blanco, para que fuera completado por un iluminador posteriormente.

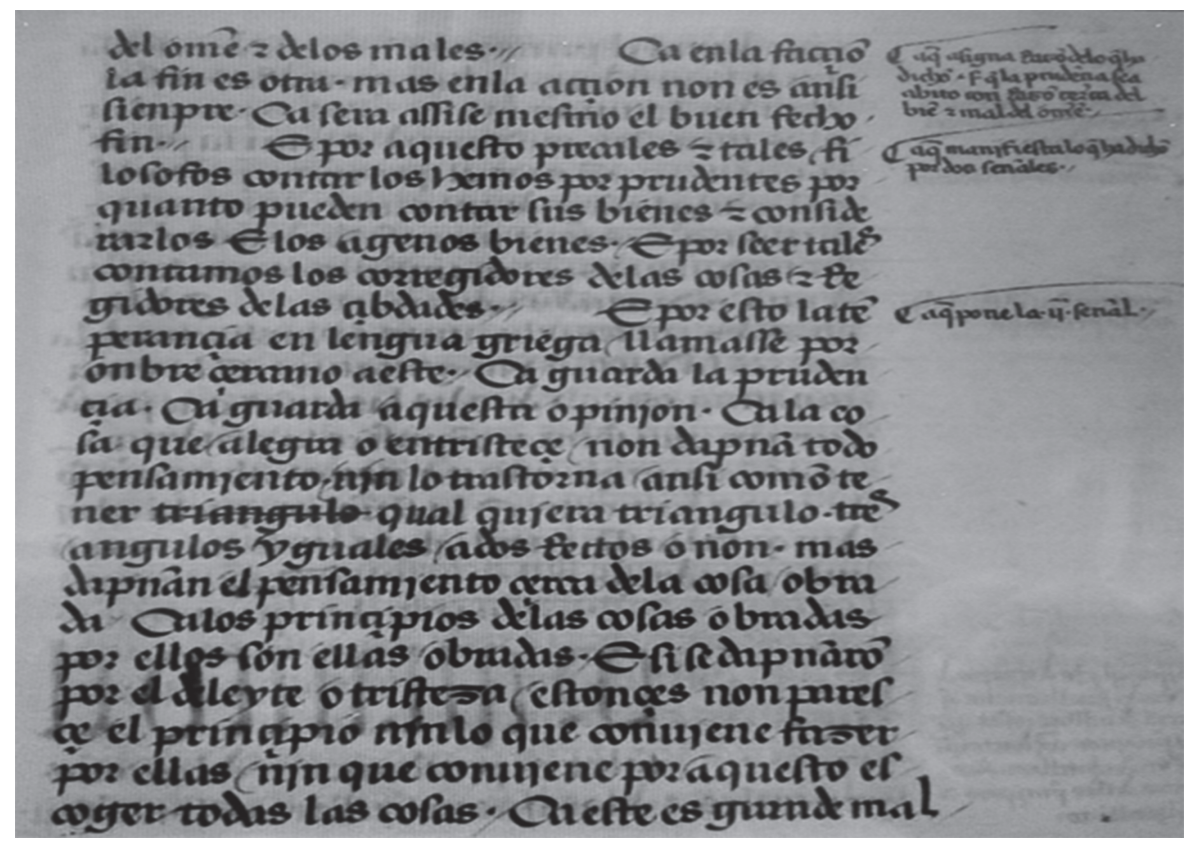

Ms. Madrid, BNE, 10268, f. 107, 1. 4.

En cuanto al paso dedicado a la explicación de la virtud de la prudencia, podemos comprobar que tampoco lee correctamente el antropónimo "Pericles" (f. 107r):

[...] E por aquesto Precales e tales filósofos contarlos hemos por prudentes, por quanto pueden contar sus bienes e considerarlos e los agenos bienes. E por seer tales contámoslos corregidores de las cosas e regidores de las cibdades. 
Una vez consignados los tres textos del primer fragmento en comparación, podemos concluir que la única traducción que reporta fielmente el nombre de Pericles es la del príncipe de Viana. En cuanto al sentido del pasaje, atestiguamos que el paso del Compendio es ininteligible al desconocer que el nombre de Pericles es el ejemplo vivo de la prudencia. En relación con el paso en la traslación del príncipe de Viana, observamos que se opta por traducir tá agathá por "aquellas cosas que fueron útiles", una solución moderna que desvirtúa la letra aristotélica. En este punto concreto, es más fiel el Ms. BNE 10268, al traducir por "bienes", como se aprecia en el párrafo que acabamos de transcribir.

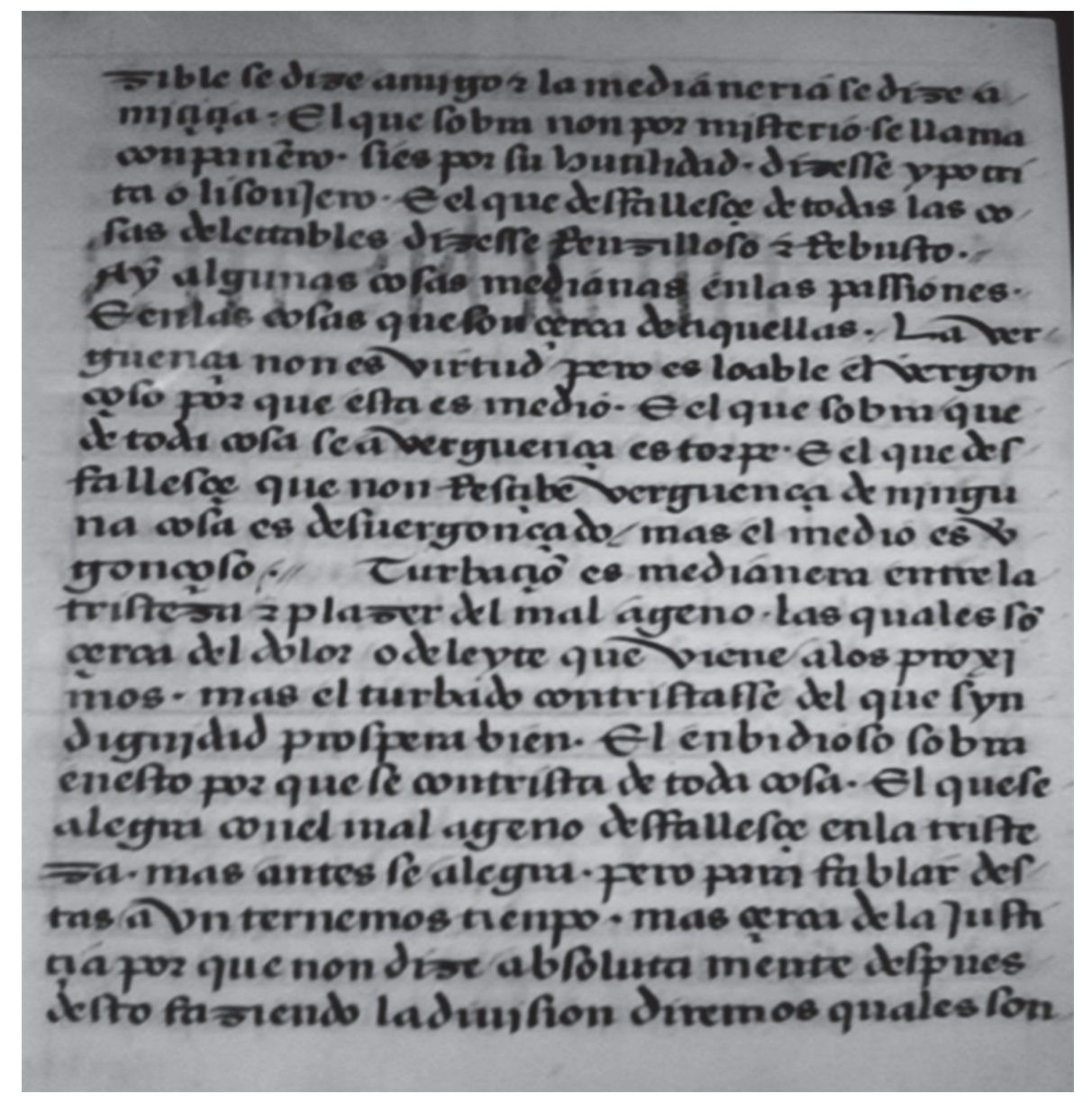

Ms. Madrid, BNE, 10268, f. 30r, 1. 13. 
En relación con el segundo fragmento, el Ms. BNE 10268 utiliza la palabra "turbación" para referirse a la virtud aristotélica de la némesis, la "indignación" — traducida correctamente por Carlos de Aragón - sentida cuando prosperan aquellos que no se lo merecen. El Compendio deformaba la transliteración del concepto griego convirtiéndola en "nemesia", una caricatura lingüística. En cuanto a los vicios que se alejan del punto medio de la justa indignación, el Ms. BNE 10268 coincide con las otras dos versiones al emplear el término "envidia" para referirse al vicio de entristecerse de la prosperidad ajena, sea cual sea, merecida o inmerecida; mientras que diverge en la traducción de la epichairekakía de marras. He aquí la transcripción de este pasaje:

Turbación es medianera entre la tristeza e plazer del mal ageno, las quales son cerca del dolor o deleyte que viene a los próximos, mas el turbado contrístasse del que syn dignidad prospera bien. El enbidioso sobra en esto, porque se contrista de toda cosa. El que se alegra con el mal ageno deffallesce en la tristeza, mas antes se alegra.

El Ms. BNE 10268 opta por el sintagma "plazer del mal ageno" para traducir el concepto epichairekakía, explicado equivocadamente como la alegría del mal ajeno, en vez de cómo el gozo de la prosperidad de los malos. En este paso, se revela la superioridad de la traducción del príncipe de Viana sobre las otras dos, ya que el Compendio pervierte el significado al tratar de simplificar la complejidad del análisis aristotélico y el Ms. BNE 10268 no acierta a explicar adecuadamente el vicio de la epichairekakía. No obstante, en su vocación simplificadora el Compendio nos proporciona una posible crítica al Estagirita lanzada desde el sentido común, ya que es la apátheia y no la epichairekakía la que parece ser el extremo por defecto de la némesis. Por lo tanto, la "no-curança" del Compendio, entendida como indiferencia, pervertiría la letra aristotélica, pero introduciría una interesante crítica a la autoridad del Philosophus, que se resquebrajaba en otros ámbitos por las dudas introducidas desde la física de Buridán, la anatomía de Vesalio o la astronomía de Copérnico.

Antes de acometer el estudio del último de los tres fragmentos, volvemos sobre el detalle de que el uso de la palabra "deleite" en detrimento del "placer", acercaría el Ms. BNE 10268 a la tradición escolástica de Alfonso de Cartagena, mientras que la traducción principesca a la corriente humanista encabezada por Leonardo Bruni. 


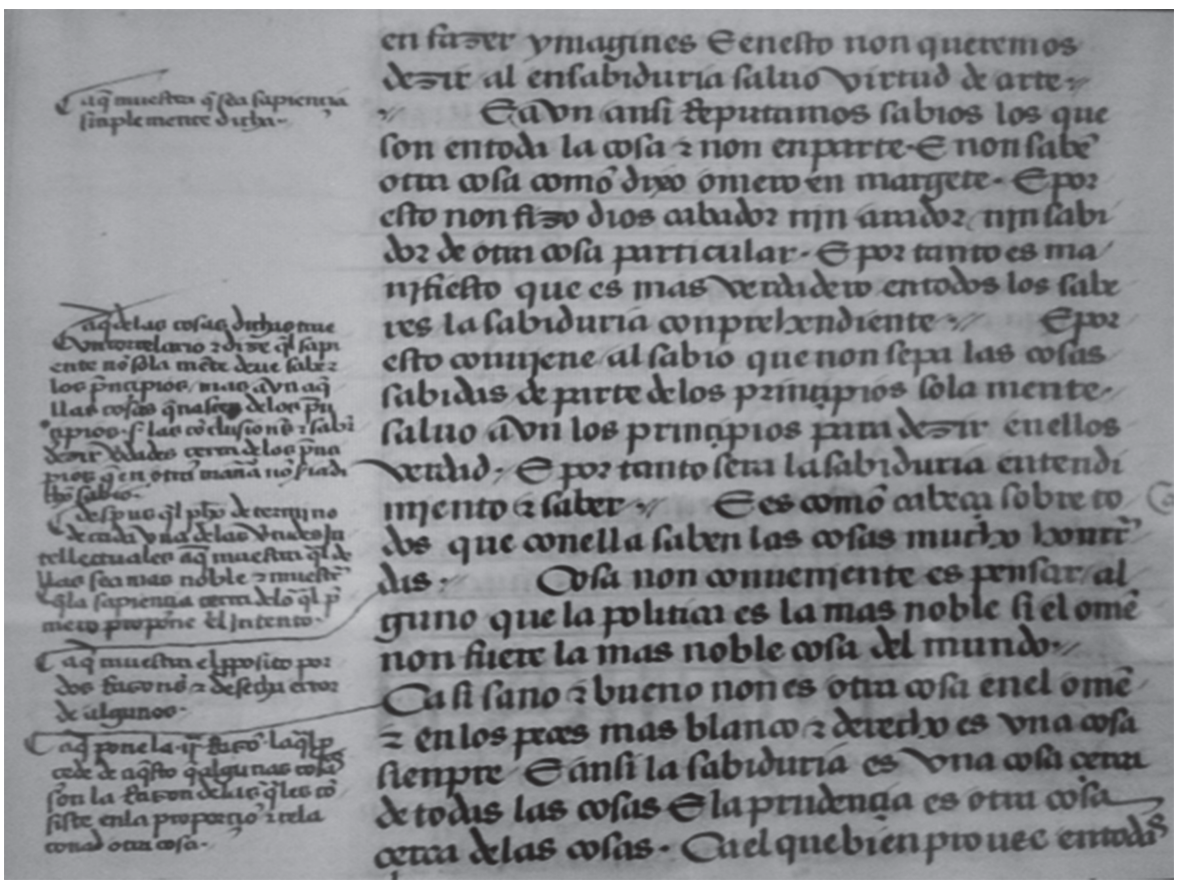

Ms. Madrid, BNE, 10268, f. 108v

En el tercer fragmento, el Ms. BNE 10268 opta por sustantivar los infinitivos "saber" y "entender" para combinarlos en la definición de la sabiduría. La sustantivación de "saber" para trasladar epistéme es problemática en el contexto de la "sabiduría", que es como traducen sophía Carlos de Viana y del Ms. BNE 10268; el Compendio, por el contrario, opta por el arcaísmo "sabieza".

En los folios siguientes al arriba consignado, el 109 recto y verso, se acumulan una serie de datos importantes para acercarnos, quinientos años después, a las características del Ms. BNE 10268. El primer detalle nos devuelve a la dificultad de leer los antropónimos griegos, ya que nos reporta los nombres de "Amángoras e Cahalis" en lugar de Anaxágoras y Tales, cosa que nos reafirma en la conclusión de que la traducción de Carlos de Viana es la óptima en este aspecto. El segundo nos sumerge nuevamente en el Leitmotiv del artículo: la irrupción de la utilidad como valor supremo del pensamiento europeo a partir del Quattrocento. El Ms. BNE 10268 había sido fiel al significado de tá agathá al traducirlo por "bienes" y no por "cosas útiles", como hace el moderno príncipe de 
Viana. En el f. 109v, el Ms. BNE 10268 reitera su compromiso con la superioridad de la sabiduría inútil al traducir que los sabios se diferenciaban del resto de la especie porque "non sabían las cosas que eran útiles a ellos"; no obstante, "sabían grandes cosas, terribles, fuertes, divinas, mas non aprovechables a otros, ca non demandavan los bienes humanos". Conviene resaltar el conflicto de valores inherente a este párrafo y que atraviesa, tal vez, toda la evolución de la ética aristotélica: "el bien en sî" contra "el bien para los hombres". Los sabios buscarían un bien inútil y sin provecho y, por ello, divino. Los prudentes se dirigirían hacia el bien para los hombres, no según lo que le parece a cada cual, sino según

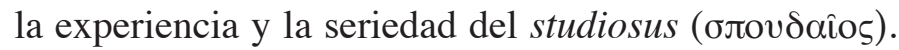

\section{Conclusiones provisorias}

a) El Compendio escolástico simplifica de tal manera el contenido de la Ética nicomaquea que hace difícil su comprensión, hasta el punto de condensar en una sola oración diversas frases aristotélicas o de suprimirlas en aras de la brevedad; por ello, ejemplificaría negativamente la hipertrofia de la traducción sententia ad sententiam. Su pertenencia a la tradición escolástica se puede demostrar por su estructura, propia del género escolar de las conclusiones, y por el uso y abuso de términos griegos transliterados, tal como se repetía desde las traducciones de Grosseteste.

b) El trabajo del príncipe de Viana muestra las características de una traducción humanista verbum e verbo, como, por ejemplo, la dignificación de la lengua de llegada. Al emplear el romance para verter con precisión los conceptos aristotélicos, se enmarcaría en un humanismo vernáculo alejado del paradigma florentino y de las directrices de utilizar exclusivamente el latín ciceroniano. Al huir de la transliteración de los conceptos griegos y cuidar la fidelidad de los antropónimos helenos, seguiría la estela de los trabajos de Bruni. Al ensalzar el valor de la utilidad, se inscribiría en la nómina de intelectuales modernos, inaugurada, entre otros, por Coluccio Salutati con su carta a fra Giovanni degli Angeli. Sin embargo, la inclusión de un aparato de conclusiones y de glosas extraídas de los Sententia de santo Tomás de Aquino, lo emparentaría con la tradición escolástica. Por tanto, el proceder de Carlos de Viana se 
podría describir con rasgos tanto de la traducción humanista, como de la tradición escolástica, evitándonos caer en la tentación de establecer rupturas totales entre etiquetas historiográficas.

c) La translación contenida en el Ms. BNE 10268 también se incluiría en la categoría de verbum e verbo; no obstante, la presencia de conceptos griegos transliterados, como "entrapella" o "bomolotos", de deformaciones de antropónimos helenos, como "Precales" o "Amángoras", y el utilizar de un vasto sistema de notas marginales, aún por estudiar, la introducirían dentro el conjunto de las traducciones escolásticas.

\section{BIBLIOGRAFÍA}

Alvar, Carlos, Traducciones y traductores. Materiales para una historia de la traducción en Castilla durante la Edad Media, Alcalá de Henares, Centro de Estudios Cervantinos, 2010 (Historia y Literatura, 2).

Aquino, Tomás de, Sententia Libri Ethicorum, ed. René-Antoine Gauthier, Roma, Comissio Leonina, 1969.

Bentley, J. H., Politics and Culture in Renaissance Naples, Princeton, Princeton University Press, 1987.

BETA (Bibliografía Española de Textos Antiguos), Ch. B. Faulhaber, A. Gómez, Ó. Perea, et al. (comps.), en PhiloBiblon <http://bancroft.berkeley.edu/philobiblon/beta_es.html/>.

BITECA (Bibliografia de Textos Antics Catalans, Valencians i Balears), Vicenç Beltran, Gemma Avenoza y Lourdes Soriano (comps.), en PhiloBiblon <http:// bancroft.berkeley.edu/philobiblon/beta_es.html/>.

CABRÉ, Míriam, “ ‘Como por los márgines del libro verá vuestra alteza': la presencia del entorno alfonsí en la traducción de la Ética de Carlos de Viana”, en Actas del VII Congreso de la AHLM, Santander, Gobierno de Cantabria, 2000, pp. 411-426.

CuencA, Salvador, "L'Ėtica Nicomaquea d'Aristòtil en un compendi català del segle Xv", Anuari de la Societat Catalana de Filosofia, XXIII, 2012, pp. 7-120.

-, "Aproximación a la relación entre los manuscritos castellanos del siglo xv del compendio de la Ética Nicomaquea", en En vulgar lengua traduzido, San Millán de la Cogolla, Cilengua (en prensa).

FAUlHABER, Charles B., "Sobre la cultura ibérica medieval: las lenguas vernáculas y la traducción”, en J. M. Lucía Megías (ed.), Actas del VI Congreso Internacional de la AHLM, Alcalá de Henares, Universidad de Alcalá de Henares, 1997, pp. 587-597.

FERNANDEZ, Jorge, “Carlos de Aragón, príncipe de Viana y su traducción de la Ética nicomaquea", Alazet, 14, 2002, pp. 223-232. 
FrANCESCHINI, Ezio, Scritti di Filologia Latina Medievale II, Padua, Antenore, 1976.

GAuthiER, R. A., "Praefatio", en Ethica Nicomachea I, Leiden-Bruselas, Brill, 1974 (Aristoteles Latinus XXVI).

Heusch, Carlos, "La Morale du Prince Charles de Viana”, Atalaya, 4, 1993, pp. 93-226.

Kristeller, Paul Oskar, Renaissance Thought and its Sources, Nueva York, Columbia University Press, 1979.

LAWRAnCE, Jeremy N. H., "Nuño de Guzmán and Early Spanish Humanism: Some Reconsiderations", Medium aevum, 51, 1982, pp. 55-85.

-, Un episodio del protohumanismo español: Tres opúsculos de Nuño de Guzmán y Gianozzo Manetti, Salamanca, Diputación de Salamanca, 1989.

LiNES, David A., Aristotle's Ethics in the Italian Renaissance (ca. 1300-1650), Leiden-Boston-Köln, Brill, 2002.

- , "Humanistic and scholastic ethics", en The Cambridge companion to Renaissance philosophy, Cambridge, Cambridge University Press, 2007, pp. 304-318.

MARTínez Romero, Tomàs, Tòpics literaris, traducció literària i tradició romànica, Barcelona, Institut d'Estudis Catalans, 2007.

Pagden, Anthony R. D., "The diffusion of Aristotle's Moral Philosophy in Spain, ca. 1400-ca. 1600", Traditio, XXXI, 1975, pp. 287-313.

RonZÓn, Elena, "El descubrimiento del hombre. Una interpretación desde la historia de la antropología filosófica", Thémata, Revista de Filosofía, 46 (Segundo semestre), 2012, pp. 217-235.

Russell, Peter y Anthony PAGDEN, "Nueva luz sobre una versión española cuatrocentista de la Ética a Nicómaco: Bodleian Library, Ms. Span. d. l", en Homenaje a Guillermo Guastavino, Madrid, Asociación Nacional de Bibliotecarios, Archiveros y Arqueólogos, 1974, pp. 125-46. 
